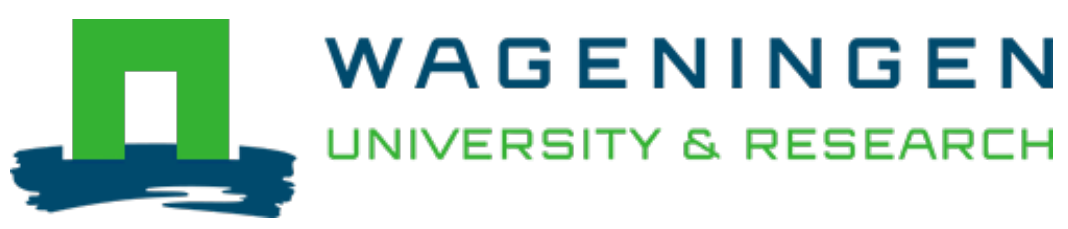

\author{
Gut bacteria formation and influencing factors \\ FEMS Microbiology Ecology \\ Yang, Jing; Wu, J.; Li, Yating; Zhang, Y.; Cho, William C. et al \\ https://doi.org/10.1093/femsec/fiab043
}

This article is made publicly available in the institutional repository of Wageningen University and Research, under the terms of article $25 \mathrm{fa}$ of the Dutch Copyright Act, also known as the Amendment Taverne. This has been done with explicit consent by the author.

Article $25 \mathrm{fa}$ states that the author of a short scientific work funded either wholly or partially by Dutch public funds is entitled to make that work publicly available for no consideration following a reasonable period of time after the work was first published, provided that clear reference is made to the source of the first publication of the work.

This publication is distributed under The Association of Universities in the Netherlands (VSNU) 'Article $25 \mathrm{fa}$

implementation' project. In this project research outputs of researchers employed by Dutch Universities that comply with the legal requirements of Article $25 \mathrm{fa}$ of the Dutch Copyright Act are distributed online and free of cost or other barriers in institutional repositories. Research outputs are distributed six months after their first online publication in the original published version and with proper attribution to the source of the original publication.

You are permitted to download and use the publication for personal purposes. All rights remain with the author(s) and / or copyright owner(s) of this work. Any use of the publication or parts of it other than authorised under article $25 \mathrm{fa}$ of the Dutch Copyright act is prohibited. Wageningen University \& Research and the author(s) of this publication shall not be held responsible or liable for any damages resulting from your (re)use of this publication.

For questions regarding the public availability of this article please contact openscience.library@wur.nl 


\title{
MINIREVIEW
}

\section{Gut bacteria formation and influencing factors}

\author{
Jing Yang ${ }^{1, \dagger}$, Jin'en $\mathrm{Wu}^{1, \dagger}$, Yating $\mathrm{Li}^{1}$, Yong'e Zhang ${ }^{1}$, William C. Cho ${ }^{2}$, \\ Xianghong Ju ${ }^{3}$, Evert M. van Schothorst ${ }^{4}$ and Yadong Zheng ${ }^{1,5, *, \ddagger}$
}

\begin{abstract}
${ }^{1}$ State Key Laboratory of Veterinary Etiological Biology, Key Laboratory of Veterinary Parasitology of Gansu Province, Lanzhou Veterinary Research Institute, CAAS, 1 Xujiaping, Chengguan District, Lanzhou 730046, China, ${ }^{2}$ Department of Clinical Oncology, Queen Elizabeth Hospital, 30 Gascoigne Road, Hong Kong SAR 999077, China, ${ }^{3}$ Department of Veterinary Medicine, College of Agriculture, Guangdong Ocean University, 1 Haida Road, Mazhang District, 524088, China, ${ }^{4}$ Human and Animal Physiology, Wageningen University, De Elst 1, Wageningen 6708WD, The Netherlands and ${ }^{5}$ Jiangsu Co-innovation Center for Prevention and Control of Important Animal Infectious Diseases and Zoonoses, 88 Daxuenan Road, Yangzhou 225009, China

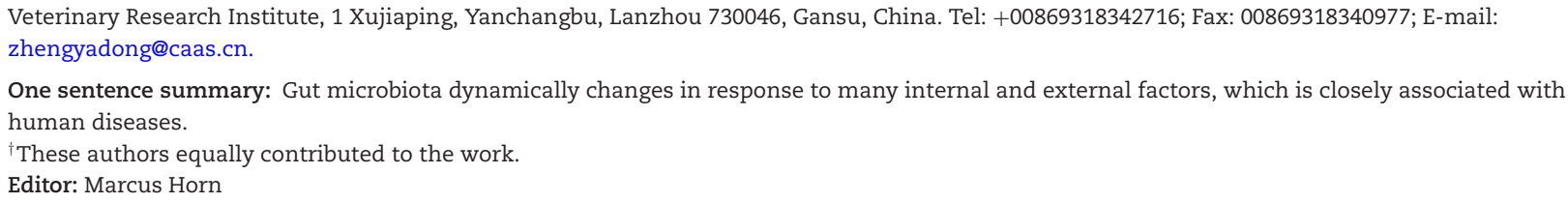

\begin{abstract}
The gut microbiota plays an important role in human health. In modern life, with the improvement of living conditions, the intake of high-sugar and high-fat diets as well as the large-scale use of antibacterial drugs have an extensive impact on the gut microbiota, even leading to gut microbiota-orchestrating disorders. This review discusses the effects of various factors, including geographic location, age, diet, antibacterial drugs, psychological situation and exercise on gut bacteria, which helps us profoundly to understand the significance of gut bacteria to human health and to find effective solutions to prevent or treat related diseases.
\end{abstract}

Keywords: gut bacteria; geographic location; age; diet; antibacterial drugs; exercise

\section{ABBREVIATIONS}

FMT:

CS:

EBF:

HMP:

OTUs:

WG:

RGs:

DF:

SCFAs $^{+}$:

whole grains;

dietary fiber; fecal microbiota transplantation;

caesarean section;

exclusively breastfed;

Human Microbiota Project;

operational taxonomic units;

refined grains;

short-chain fatty acids;
ICIs: Immune Checkpoint Inhibitors;

T1D: $\quad$ type 1 diabetes;

T2D: $\quad$ type 2 diabetes;

PD-1: $\quad$ programmed cell death protein 1;

PD-L1: $\quad$ programmed cell death protein ligand 1;

JAX: Jackson Laboratory (JAX);

TAC: $\quad$ Taconic Farms;

$\mathrm{mAbs}^{+}$: monoclonal antibodies;

PIs: $\quad$ proton pump inhibitors;

AAPs: atypical antipsychotics;

HPA: hypothalamic-pituitary-adrenal;

Received: 27 August 2020; Accepted: 9 March 2021

(C) The Author(s) 2021. Published by Oxford University Press on behalf of FEMS. All rights reserved. For permissions, please e-mail: journals.permissions@oup.com 
CNS: $\quad$ central nervous system;

IBD: inflammatory bowel disease;

IBS: irritable bowel syndrome;

IL-1 $\beta$ : leukocyte-1 $\beta$;

IDO1: indoleamine 2,3 dioxygenase;

IL-23: interleukin-23;

MI: myocardial infarction;

CVD: $\quad$ cardiovascular diseases;

CD: Crohn disease;

UC: $\quad$ Ulcerative Colitis;

rRNA: ribosomal RNA;

CDI: Clostridium difficile infection

\section{INTRODUCTION}

The intestine is the main site for microbiota colonization in the body. Typically, when children grow to 3 years old, their gut microbiota compositions are close to the adult's, being a relative stable gastrointestinal microbiota with more than 500 different microbial species (Tang, Li and Hazen 2019). In contemporary world, the economic, social, technological progresses have transformed human lifestyles, and most people in the developed countries has enriched material life. Interestingly, the gut microbiota has also changed and these changes directly or indirectly relate to human multifactorial diseases (Levy et al. 2017), such as obesity (Maruvada et al. 2017), diabetes (Brunkwall and Orho-Melander 2017), neurodegeneration (Dinan and Cryan 2017), allergy (McKenzie et al. 2017) and asthma (Guilleminault et al. 2017; Levy et al. 2017; Maruvada et al. 2017). In Africa, the adoption of western lifestyle and dietary habits also exacerbates the risk of noncommunicable diseases, such as cancer, cardiovascular disease (CVD), and type 2 diabetes (T2D) (Brewster et al. 2019). However, the role of the gut microbiota to these diseases remains unclear. But it seems clear that the extensive use of antibiotics has led to colonization resistance loss and antibiotics-induced dysbiosis of intestinal microbiota (Kim, Covington and Pamer 2017). When the gut microbiota is dysfunctional, the proportion of beneficial bacteria is reduced with the increased proportion of harmful bacteria. This imbalance may affect human health in various aspects, including the production of harmful substances such as endotoxin and ammonia, abnormal functions of intestinal peristalsis and hypoimmunity. Dealing with these health issues, there is increasing evidence that fecal microbiota transplantation (FMT) is a promising way to restore a normal microbiota and thus cure the disease. It is worthy to mention that FMT is still a black box with unknown risks and side effects, especially some particular metabolites or drugs in the feces that adversely affect the recipient patients. For instance, valerate is a metabolite that may be a critical determinant in treating recurrent Clostridioides difficile infection (CDI) via FMT, but its minimum dose and mechanism have not been determined (Cresci and Bawden 2015; Bibbo et al. 2017; Vindigni and Surawicz 2017; McDonald et al. 2018; Mullish et al. 2018). Although the relationship between gut microbes and diseases has drawn great attention, many issues are yet to be addressed. These include how to identify beneficial bacterial species and select potential immunomodulatory metabolites from dietary constituents (Wypych, Marsland and Ubags 2017), and whether there is a causal relationship between dysbiosis of gut flora and occurrence of autoimmune diseases (Bach 2018).

The gastrointestinal tract is a major site of microbial colonization and growth (Riaz Rajoka et al. 2018). Although humans form a stable intestinal flora at the age of 3 , this relative stability is affected by the following factors in a different way, including geographic location, age, diet, antibacterial agents, psychological situation, stress and exercise (Cresci and Bawden 2015; Gupta, Cifu and Khanna 2018). Moreover, in response to different influencing factors there are different dominant or low abundance bacteria being observed (Table 1). Although the terms microbiota, the bacteria, archaea, protists, fungi and viruses and microbiome, the bugs and their genes, are often used synonymously, they in fact represent slightly different entities. Here, we focus our review mainly on bacteria.

\section{FORMATION OF GUT MICROBIOTA}

Initially, it is believed that the fetus is sterile in the uterus, and the first colonization of the intestinal flora occurs during the giving-birth process (Salazar et al. 2014). This traditional understanding had been revolutionized following the discovery of microorganisms in amniotic fluid, the umbilical cord, placenta and preterm infant meconium (Cresci and Bawden 2015). The process from birth to the formation of adult-like gastrointestinal microbiota is influenced by many determinants, as summarized in Fig. 1.

The human gut serves as an essential habitat for a diverse microbiota ecosystem. At birth, when infants are continuously exposed to the external environment, the intestines are quickly colonized by trillions of microbes $\left(10^{13}-10^{15}\right)$ (Tang, Li and Hazen 2018). The early establishment of gut microbiota is important for subsequent health (Rautava 2016; Ho et al. 2018), with gut microbiota compositions showing differences by delivery mode (Munyaka, Khafipour and Ghia 2014; Cresci and Bawden 2015; Rutayisire et al. 2016). It was manifested that caesarean section (CS) had the higher abundance and diversity of the Phylum Firmicute (Clostridium and Lactobacilli), but at the colonization level, Bifdobacterium and Bacteroides genera were significantly more frequent in vaginally delivered infants than CS infants (Adlerberth et al. 2007; Rutayisire et al. 2016). However, this diversity and colonization pattern of the gut microbiota are only significantly associated with the mode of delivery in the first three months of life, and this difference gradually disappears within 6 months after birth.

After birth, the intestinal microbes of an infant are mainly from the mother's skin, breastfeeding and/or formula feeding (Pannaraj et al. 2017; Ho et al. 2018), and 25\%-30\% of the bacterial microbiota is derived from breast milk (Pannaraj et al. 2017; Robertson et al. 2019). Breast milk is considered the golden standard for infants, but it is not sterile and contains about 600 different bacteria species including Bifidobacterium species, such as Bifidobacterium breve, Bifidobacterium adolescentis, Bifidobacterium longum, Bifidobacterium bifidum, and Bifidobacterium dentium (Mohammadkhah et al. 2018). In breast milk, oligosaccharides have been reported to play an essential role in promoting the colonization of microorganisms and are beneficial to the growth of microorganisms, especially Bifidobacteria (Charbonneau et al. 2016; Riaz Rajoka et al. 2018). Infants exclusively breastfed (EBF) and non-EBF were significantly different in their aspects of intestinal microbial diversity, microbiota age, microbial compositions and functions in the first 6 months after birth (Cresci and Bawden 2015; Pannaraj et al. 2017; Ho et al. 2018). After 6 months of age, these differences sustain, and EBF infants have a shorter duration of having higher abundance in Lactobacillaceae, Coriobacteriaceae, Prevotellaceae, Clostridiaceae, Erysipelotrichaceae and Lachnospiraceae, and lower abundance in Bifidobacteriaceae and Enterococcaceae (Ho et al. 2018). 
Table 1. The relative beneficial or harmful bacteria in the gut microbiota in response to influencing factors.

\begin{tabular}{|c|c|c|c|c|}
\hline Influencing factors & & Beneficial/dominant bacteria & Harmful/low abundance bacteria & References \\
\hline \multirow[t]{5}{*}{ Geographic location } & Japan & $\begin{array}{l}\text { Bacteroidetes, Firmicutes, } \\
\text { Actinobacteria, Bifidobacterium }\end{array}$ & Clostridium, Alistipes & $\begin{array}{l}\text { (Nishijima et al. 2016; } \\
\text { Mano et al. 2018) }\end{array}$ \\
\hline & China & $\begin{array}{l}\text { Bacteroidetes, Proteobacteria, } \\
\text { Firmicutes, Actinobacteria }\end{array}$ & $\begin{array}{l}\text { Megamonas, Succinivibrio, } \\
\text { Prevotella }\end{array}$ & $\begin{array}{l}\text { (Kuang et al. 2016; Liao } \\
\text { et al. 2018) }\end{array}$ \\
\hline & India & $\begin{array}{l}\text { Bacteroidetes, Firmicutes, } \\
\text { Proteobacteria, Actinobacteria, } \\
\text { Prevotella }\end{array}$ & / & $\begin{array}{l}\text { (Kumbhare et al. 2017; } \\
\text { Brewster et al. 2019; } \\
\text { Tandon et al. 2018) }\end{array}$ \\
\hline & Argentinan & $\begin{array}{l}\text { Ruminococcaceae, Lachnospiraceae, } \\
\text { Rikenellaceae, Prevotellaceae }\end{array}$ & / & (Carbonetto et al. 2016) \\
\hline & USA & $\begin{array}{l}\text { Ruminococcaceae, Lachnospiraceae, } \\
\text { Rikenellaceae Prevotellaceae }\end{array}$ & / & $\begin{array}{l}\text { (Carbonetto et al. 2016; } \\
\text { Hansen et al. 2019) }\end{array}$ \\
\hline \multirow[t]{4}{*}{ Age } & Infants & $\begin{array}{l}\text { Bifidobaterium, Fusobacteria, } \\
\text { Proteobacteria }\end{array}$ & $\begin{array}{l}\text { Enterococcus faecalis, } \\
\text { Methanobrevibacter }\end{array}$ & $\begin{array}{l}\text { (Cresci and Bawden 2015; } \\
\quad \text { Milani et al. 2017) }\end{array}$ \\
\hline & Adults & $\begin{array}{l}\text { Firmicutes, Bacteroidetes, } \\
\text { Proteobacteria, Fusobacteria, } \\
\text { Cyanobacteria, Verrucomicrobia }\end{array}$ & $\begin{array}{l}\text { Bifidobacteria, Clostridium cluster } \\
\text { XIVa }\end{array}$ & $\begin{array}{l}\text { (Ottman et al. 2012; } \\
\text { Salazar et al. 2017) }\end{array}$ \\
\hline & The elderly (>65) & $\begin{array}{l}\text { Bacteroidetes, Clostridia, } \\
\text { Lactobacilli, Streptococci, } \\
\text { Enterobacteriaceae, Proteobacteria, } \\
\text { Escherichia coli }\end{array}$ & $\begin{array}{l}\text { Firmicutes, Clostridium XIVa, } \\
\text { Faecalibacterium prausnitzii, } \\
\text { Actinobacteria }\end{array}$ & $\begin{array}{l}\text { (O'Toole and Jeffery 2015; } \\
\text { Salazar et al. 2017; An } \\
\quad \text { et al. 2018) }\end{array}$ \\
\hline & Centenarians & $\begin{array}{l}\text { Akkermansia, Clostridium XIVa, } \\
\text { Bifidobacterium }\end{array}$ & $\begin{array}{l}\text { Faecalibacterium, Roseburia, } \\
\text { Coprococcus, Blautia, }\end{array}$ & $\begin{array}{l}\text { (Biagi et al. 2016; Kong } \\
\text { et al. 2016; Biagi et al. } \\
\text { 2017; Kong et al. 2018) }\end{array}$ \\
\hline \multirow[t]{5}{*}{ Diet } & Thai vegetarians & $\begin{array}{l}\text { Prevotella copri, Faecalibacterium } \\
\text { prausnitzii }\end{array}$ & $\begin{array}{l}\text { Alistipes putrednis, Bilophila } \\
\text { wadsworthia }\end{array}$ & $\begin{array}{l}\text { (Ruengsomwong et al. } \\
\text { 2016) }\end{array}$ \\
\hline & $\begin{array}{c}\text { Thai } \\
\text { non-vegetarians }\end{array}$ & $\begin{array}{l}\text { Bacteroides vulgatus, } \\
\text { Faecalibacterium prausnitzii }\end{array}$ & $\begin{array}{l}\text { Bilophila wadsworthia, Coprococcus } \\
\text { eutactus }\end{array}$ & $\begin{array}{l}\text { (Ruengsomwong et al. } \\
\text { 2016) }\end{array}$ \\
\hline & WGs & Lachnospira, Roseburia & Enterobacteriaceae & (Vanegas et al. 2017) \\
\hline & $\mathrm{DF}$ and prebiotic & $\begin{array}{l}\text { Actinomycetes, Bifidobacteriaceae, } \\
\text { Lactobacilli }\end{array}$ & $\begin{array}{l}\text { Ruminococcus, Lachnobacterium, } \\
\text { Anaerostipes, Ruminococcus, } \\
\text { Escherichia coli }\end{array}$ & $\begin{array}{l}\text { (Holscher 2017; Mano } \\
\text { et al. 2018) }\end{array}$ \\
\hline & Mediterranean diet & $\begin{array}{l}\text { Bacteroidetes, Roseburia, } \\
\text { Lachnospira, Prevotella, Firmicutes, } \\
\text { Candida }\end{array}$ & Escherichia coli & $\begin{array}{l}\text { (De Filippis et al. 2016; } \\
\text { Mitsou et al. 2017; Ni } \\
\text { Lochlainn, Bowyer and } \\
\text { Steves 2018) }\end{array}$ \\
\hline Antibacterial drugs & Antibiotics & Bacteroidetes, Proteobacteria & $\begin{array}{l}\text { Bifidobacterium, Akkermansia } \\
\text { muciniphila, Lactobacilli }\end{array}$ & $\begin{array}{l}\text { (Sivan et al. 2015; Ianiro, } \\
\text { Tilg and Gasbarrini 2016; } \\
\text { Routy et al. 2018) }\end{array}$ \\
\hline \multirow[t]{2}{*}{$\begin{array}{l}\text { Psychological } \\
\text { situation and stress }\end{array}$} & Depression, anxiety & $\begin{array}{l}\text { Enterobacteriaceae, Alistipes, } \\
\text { Bacteroidales }\end{array}$ & Faecalibacterium, Lachnospiraceae & (Jiang et al. 2015; Liu 2017) \\
\hline & $\begin{array}{l}\text { Psychological stress } \\
\text { and social stress }\end{array}$ & Firmicutes, Clostridium & $\begin{array}{l}\text { Lactobacillus spp, Bifidobacteria, } \\
\text { Lactobacilli, Alistipes }\end{array}$ & (Liu 2017; Lach et al. 2018) \\
\hline \multirow[t]{3}{*}{ Exercise } & Competitive cyclists & $\begin{array}{l}\text { Prevotella, Methanobrevibacter } \\
\text { smithii, Bacteroidetes, Firmicutes, } \\
\text { Euryarchaeota }\end{array}$ & $\begin{array}{l}\text { Achromobacter, Brachymonas, } \\
\text { Candidatus, Desulfobulbus, } \\
\text { Leptothrix }\end{array}$ & $\begin{array}{l}\text { (Petersen et al. 2017; Allen } \\
\text { et al. 2018) }\end{array}$ \\
\hline & Rugby athletes & Akkermansia muciniphila & Bacteroide, Lactobacillus & $\begin{array}{l}\text { (Petersen et al. 2017; } \\
\text { Barton et al. 2018) }\end{array}$ \\
\hline & $\begin{array}{l}\text { Elite athletes, } \\
\text { rowers }\end{array}$ & Veillonella & - & (Scheiman et al. 2019) \\
\hline
\end{tabular}

In humans at age of 3 , the composition of gut microbiota remains relatively stable over time with introduction of solid food (Cresci and Bawden 2015; Liu et al. 2016; Ho et al. 2018; Mohammadkhah et al. 2018). Nevertheless, throughout their life, humans are exposed to a variety of living conditions, especially taking high-fat diets and antibacterial drugs, and as consequences, the gut microbiota may develop into the direction being healthy or detrimental to the body (Salazar et al. 2014). Thus, the formation of gut microbiota undergoes a long period from the first establishment in new-born babies to the stability in children, and this relative stability is dynamically fluctuated and even disrupted by external factors.

\section{INFLUENCING ELEMENTS OF HUMAN GUT MICROBIOTA}

The gut microbes are increasingly considered to have a fundamental role in human physiology and health (Rothschild et al. 2018). Under normal circumstances, intestinal microbes are in equilibrium and coexist with the host in a mutualism way. The human gut provides the habitat and nutrients needed for gut microbiota growth, and the gut microbiota in turn provides substrates, enzymes, vitamins and energy for human metabolic processes (Arumugam et al. 2011). Simultaneously, the short chain fatty acids (SCFAs) produced by microbial metabolism 


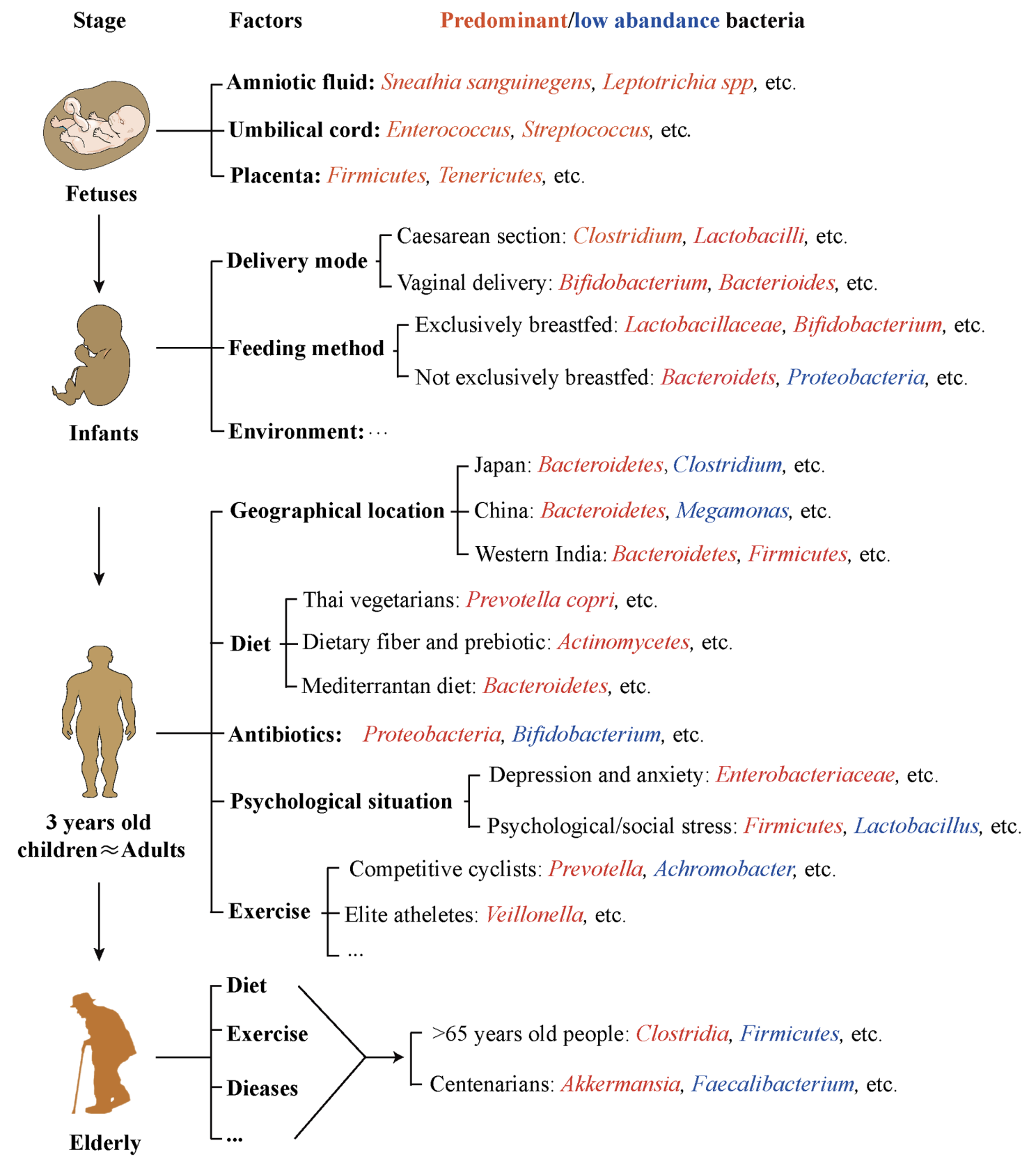

Figure 1. The influencing factors of gut bacteria formation in humans. Different influencing factors are involved in the gut bacteria formation in humans from the fetuses to the elderly. In humans at age of 3 , the composition of gut microbiota becomes relatively stable over time and is similar to the adult's. In response to the influencing factors, some bacteria become predominant (in red), but some become lowly abundant (in blue).

promote the growth and differentiation of human epithelial cells and participate in the synthesis of vitamins and the absorption of various ions. The intestine is considered to be the largest immune organ in the body. The communication between the intestinal microbes and the host on the surface of the intestinal mucosa also promotes the establishment and development of the immune system. For example, the robust enterohaemorrhagic Escherichia coli colonization in the mammalian intestine relies on the fucose-sensing system to modulate the pathogenicity and virulence gene expression (Pacheco et al. 2012). Therefore, the gut microbiota may be one of the standards for characterizing the healthy state of the body (Kong et al. 2018).

\section{Geographical location}

The geographical location affects the diversity and composition of gut microbiota in infants and adults (Table 1) (Cresci and Bawden 2015). The geographical specificity may imply that it is impossible to have a universal 'healthy microbiota'. A study in different geographical populations supported the idea of the differences in intestinal microbiota compositions in people living in different continents (Magne et al. 2016). For example, the Phyla Bacteroidetes, Firmicutes and Actinobacteria are the main three kinds of gut bacteria in Japanese, partially due to their staple food structure (Mano et al. 2018). In China, Yao populations, who are a relatively isolated minority and live in the 
heart of the forest, have a unique genus Megamonas compared to the local minority Zhuang and rural Han people. Unlike the Japanese, the dominant bacteria of these three groups are the genus Bacteroides and Prevotella (Liao et al. 2018). With the urbanization of China, the higher abundance of microbes in the urban population is the same as Americans', while archaea and viruses are gradually lost compared to the rural population in China (Winglee et al. 2017). This phenomenon may be attributed to geographical distribution and the changes in foods and environment. In western India, the gut microbiota in an adult urban population mainly consisted of four phyla, Bacteroidetes (71.5\%), Firmicutes (18.7\%), Proteobacteria (3.8\%) and Actinobacteria (0.6\%) (Tandon et al. 2018). Similarly, Latin Americans are significantly different in gut microbiota from those who are from other continents. For instance, Firmicutes are decreased but Bacteroidetes have no changes in Colombian adults compared with Americans, Europeans and Asians (Escobar et al. 2014; Magne et al. 2016). Moreover, Ruminococcaceae, Lachnospiraceae, Rikenellaceae and Prevotellaceae are more abundant in Argentinan than Americans (Carbonetto et al. 2016; Hansen et al. 2019).

To further evaluate the geographical effects, the gut microbiota composition in different origin populations was measured based on $\beta$ diversity analysis and showed that Europeans were significantly different from Indians (Rehman et al. 2016). Even for people who live in geographically adjacent places, their gut microbiota share a common diversity index and similar $\alpha$ diversity (the diversity within single sample), as evidenced by that the observed number of operational taxonomic units (OTUs) is dramatically higher in the rural Bassa infants than urban infants and adults (Ayeni et al. 2018). Therefore, geographical location as an influencing factor should be taken into account in the evaluation of the composition and diversity of the gut microbiota.

\section{Age}

Due to the introduction of solid foods, the structural and functional diversity of the infant microbiota is rapidly increased (Table 1). Once forming a microbial structure like the adult's, it remains relatively stable until old age (Cresci and Bawden 2015; Robertson et al. 2019). In terms of gut microbiota in infants, Bifidobaterium resides as the most dominant, and as time goes, the phyla Firmicutes and Bacteroidetes occupy the dominant part in adults, while Bacteroidetes become a relatively predominating proportion in the elderly (generally $>65$ years old), with an increase of Clostridia, Lactobacilli, Streptococci and Enterobacteriaceae (Ottman et al. 2012; Cresci and Bawden 2015; O'Toole and Jeffery 2015; An et al. 2018). It is noted that these results are derived from fecal samples mostly representing the end of the colon but not the entire gut. In addition to the factor of age, this differential distribution of gut microbiota may be also attributed to the external factors such as diet and exercise (O'Toole and Jeffery 2015).

The intestinal microbiota consist of various phyla that contain over 1000 different microbial species (Riaz Rajoka et al. 2018). Based on the cultivation and classical 16S ribosomal RNA (rRNA) gene approach, $\alpha$ diversity was found to decline during senescence, but this finding was not further determined by other methods, such as high-throughput 16S rRNA gene sequencing and phylogenetic microarray analysis (An et al. 2018). Therefore, the complex relationship of microbial changes with age needs to be systematically investigated. In addition, some studies have found that the Alzheimer's type dementia and frailty in the elderly are related to their own fecal microbiota imbalance, and the increased frailty has been shown to be associated with reduced $\alpha$ diversity (Bian et al. 2017; An et al. 2018). The longevity
( $>90$ years old) with a healthy state may be associated with a similar composition of fecal microbiota as seen in healthy young people (O'Toole and Jeffery 2015; Ticinesi, Tana and Nouvenne 2019). However, the diversity of the gut microbiota is greater in longevity people than young adults (Kong et al. 2018). Centenarians are shown to have the ability to delay or avoid chronic diseases. These may be attributed to some beneficial bacterial taxa (e.g. Akkermansia and Clostridium XIVa), which are known as SCFA producers enriched in the long-living people in China and Italy (Kong et al. 2016; Kong et al. 2018). However, the mechanisms by which age changes affect gut microbiota remain unclear, and need to be explored in the broader context of the genetic and lifestyle changes that accompany aging (Fontana and Partridge 2015; O’Toole and Jeffery 2015).

\section{Diet}

It is now becoming clear that diet is a major driving force of microbial dynamics (Gibbons et al. 2017). The long- and shortterm changes in dietary composition are effective in changing the structure and activity of gut microbiota (Table 1) (David et al. 2014; Lam, Zhang and Zhao 2018). The dominant flora in the human gut is related to the ratio of microbiota-accessible carbohydrates, dietary fats and proteins in the dietary structure. The restriction of microbiota-accessible carbohydrates leads not only to bacterial diversity loss but also to SCFA reduction, which eventually affect many physiological processes including energy homeostasis, lipid metabolism and inflammation. Total dietary proteins and amino acid compositions are also influencing factors for SCFA production, but the molecular mechanism is still not fully understood (Gentile and Weir 2018). Consistent with this idea, there is a significant difference in the gut microbiota of vegetarians versus non-vegetarians. In the gut microbiota of healthy Thais, the strong species indicator of vegetarians was Prevotella copri, while the species indicator of non-vegetarians was Bacteroides vulgatus (Ruengsomwong et al. 2016). Furthermore, the effects of a diet enriched with whole grains (WGs) versus refined grains (RGs) on gut microbiota are also remarkable. There was a significant decrease in Enterobacteriaceae in the WGs group versus the RGs group, but at the genus level, the Lachnospira abundance was increased in WGs group compared with the RGs group (Vanegas et al. 2017).

In addition, dietary fiber (DF) intake also contributes to the changes of intestinal microbes. SCFAs produced by DF fermentation enhance intestinal barrier functions by increasing intestinal cell proliferation and differentiation (Kieffer, Martin and Adams 2016). People who have a dietary pattern of eating rice as staple food can ingest higher DF-containing food compared to those who take edible wheat flour products as staple food (BatresMarquez, Jensen and Upton 2009). In a recent study, according to the intake of staple rice and white bread, Japanese subjects were divided into two groups, and then cross-over experiments were conducted to exchange staple food. It was found that the staple food change made Actinomycetes and Bifidobacteriaceae significantly higher after the bread period than the rice period (Mano et al. 2018).

Furthermore, some people have special habits and customs, such as smoking and alcohol consumption that also contribute to the gut microbiome dysbiosis. It has reported that people who smoke have an increased CDI rate compared than those who never smoke, and people who drink alcohol have the depletion of anti-inflammatory bacteria (Firmicutes, Pediococcus, Lactobacillus and Leuconostoc), eventually leading to intestinal leakage (Capurso and Lahner 2017). 
It is well known that dietary habit varies from one place to another around the world, so it is unlikely that a universal dietary habit or structure that is generally beneficial to the health of the intestine exists. However, there is a fairly popular diet model, the Mediterranean diet, which is considered to be beneficial to gut microbiota and is associated with lower incidence of common chronic diseases (Bowyer et al. 2018; Pignanelli et al. 2018). This diet is characterized by high intake of whole grains, vegetables, beans, fruits, unsaturated lipids and fish, by low intake of saturated fats, meat and dairy products, and by moderate drinking (Buford 2017; Mitsou et al. 2017; Barton et al. 2018; Pignanelli et al. 2018).

All in all, different diets have different effects on the structure, composition and function of intestinal microbial flora. Indeed, in a highly controlled mouse nutritional study, differences only in starch composition, not starch quantity, gave rise to an altered microbiota composition (Fernandez-Calleja et al. 2018). Therefore, the establishment of the plasticity of gut microbes is a promising strategy to prevent and cure related diseases, but it mainly relies on long-term dietary patterns and stable microbiota conformations instead of short-term diet interventions.

\section{Antibiotics and non-antibiotic drugs}

Generally, the intestinal microbial flora is relatively stable and robust, and after a small range of disturbances, it will return to normal status unless external factors endure for a long time. High frequent use of drugs like microbiota's nemesis-antibiotics often disrupts the intestinal microbiota balance and destroys the cross-talks between microbiota and immune fitness (Table 1) (Botticelli et al. 2017). Moreover, allergic and autoimmune diseases are also largely attributed to the use of antibiotics in early life, such as type 1 or 2 diabetes (T1D or T2D) (Cox et al. 2014; Candon et al. 2015; Tai, Wong and Wen 2015), asthma (Stokholm et al. 2014), CVD, cancer and obesity (Iizumi et al. 2017; Yi et al. 2018).

Recently, immune checkpoint inhibitors (ICIs) were recognized as a greatly efficient target to improve the survival of patients who suffered from metastatic melanoma, lung carcinoma, renal cell carcinoma and lymphoma (Botticelli et al. 2017). This idea has shifted attention from the tumor itself to the patient's immune system and multiple intersecting immune regulatory networks, such as the PD-1/PD-L1 axis (programmed cell death protein 1/programmed cell death protein ligand 1) (Botticelli et al. 2017; Routy et al. 2018). It has been shown that there are only $20 \%-30 \%$ clinical benefits, and its efficiency will be largely reduced because of the use of antibiotics (Elkrief et al. 2018; Soularue et al. 2018). PD-1 based immunotherapy is influenced by gut microbiota, such as commensal Bifidobacterium (Sivan et al. 2015) and Akkermansia muciniphila (Routy et al. 2018). In genetically similar, but not the same, C57BL/6 mice from Jackson Laboratory (JAX) and Taconic Farms (TAC), oral administration of JAX fecal suspension to TAC mice enhanced spontaneous antitumor immunity (making tumor growth significantly slower), which was consistent with $\alpha$ PD-L1 monoclonal antibody $(\mathrm{mAb})$ therapy and combination therapy of JAX fecal transfer and $\alpha \mathrm{PD}-\mathrm{L} 1 \mathrm{mAb}$. This antitumor effect was mainly attributed to commensal Bifidobacterium (Sivan et al. 2015). It was also shown that antibiotics decreased the clinical benefit of ICIs in advanced cancer patients, and FMT from cancer patients who responded to ICIs improved the PD-1 blockade antitumor functions. Further analysis demonstrated that oral supplementation with Akkermansia muciniphila restored the efficacy of
PD-1 blockade in antibiotics-treated mice that did not respond to ICIs. This restoration was in an interleukin-12-dependent manner by increasing the recruitment of CCR9 ${ }^{+} \mathrm{CXCR} 3^{+} \mathrm{CD} 4^{+} \mathrm{T}$ lymphocytes (Routy et al. 2018). Therefore, maintaining a healthy gut microbial flora can support patients in their treatment of cancer.

It is also noted that non-antibiotic drugs, such as antidiabetics like metformin (Forslund et al. 2017) and proton pump inhibitors (PPIs) (Jackson et al. 2016), inhibit the growth of at least one bacterial strain, which in turn regulates the efficacy and toxicity of the drug (Maier et al. 2018). In T2D patients receiving metformin treatment, E. coli was significantly increased and Intestinibacter decreased compared with the untreated group. Consistently, subsequent preclinical experiments showed that the blood glucose levels were reduced in the germ-free FMT mice and the gastrointestinal side effects were mainly caused by an increase of E. coli species. Unlike metformin, PPI contributes to the reduction of commensal bacteria diversity, leading to a decreased colonisation resistance to intestinal infections (including Clostridium difficile, Campylobacter and Salmonella) (Weersma, Zhernakova and Fu 2020). In summary, both antibiotics and non-antibiotic drugs affect the gut microbiome via different mechanisms and ultimately influence health outcomes.

\section{Psychological situation and stress}

Stress is an overall response to environmental needs or pressures in organisms. Mental stress can cause a series of physiological changes in the gastrointestinal tract, including gut motility, visceral perception, gastrointestinal secretion and intestinal permeability, which lead to activation of the hypothalamicpituitary-adrenal (HPA) axis, decrease of mucosal immune functions and alteration of the release of duodenal bicarbonate and hormones (Cresci and Bawden 2015). These changes will alter the gut microbial composition through the gut-brain axis, a bidirectional communication between the gut microbiota and the central nervous system (CNS) (Dinan and Cryan 2017b; Bercik, Collins and Verdu 2012; Cresci and Bawden 2015; Tetel et al. 2018). Accordingly, early life stress can lead to disability and prevalence of stress-related mental illnesses (such as anxiety and depression) and gastrointestinal diseases (such as inflammatory bowel disease (IBD)) and irritable bowel syndrome (IBS) (de Weerth 2017). Although there is limited evidence to demonstrate that it causes a rising trend of IBD, stress has an effect on the symptom development of IBD (Bernstein 2017). When rodents have the symptoms and behaviors of anxiety, their jejunum showed an increased abundance in Campylobacter and Citrobacter (Bruce-Keller et al. 2015). Similarly, patients suffering from depression also have higher levels of Enterobacteriaceae and Alistipes compared with healthy adults (Jiang et al. 2015). However, it has been demonstrated that Bifidobacterium breve A-1 has a potential role in improving anxiety and depressive symptoms in patients (Okubo et al. 2018). These alterations of the gut microbial composition by psychological and social stress are related to not only changes of cytokines but also an increased level of catecholamines (Table 1) (Foster and McVey Neufeld 2013; de Weerth 2017; Liu 2017).

In turn, the intestinal microbiota also affects the psychological situation, and gut microbial disorders lead to the risk of mental illness, especially depression and anxiety (Liu 2017). It has been shown that the transfer of fecal materials from natural stress-sensitive mice to non-anxiety mice triggers an anxiety-like phenotype. Conversely, the transfer of bacteria from non-anxiety mice to recipients with natural anxiety leads to a 
decrease in anxiety (Bercik et al. 2011; Sarkar et al. 2018). However, there have so far been no reports using fecal transplantation for treating anxiety and depression in humans (Sarkar et al. 2018).

\section{Physical exercise}

It is well known that physical exercise promotes the metabolism and immunity ability of the human body (Kurilshikov et al. 2017; Petersen et al. 2017; Allen et al. 2018; Song and Chan 2018), though it might also adversely effect intestinal permeability (JanssenDuijghuijsen et al. 2016). A recent study showed that physical exercise affect the health of the gut flora by altering the composition of the gut microbiota with an increased proportion of microorganisms that contribute to intestinal health (Okubo et al. 2018; Scheiman et al. 2019). Physical exercise endurance was shown to be enhanced in mice and elite athletes, being attributable to relatively increased abundance of Veillonella. This kind of bacteria contribute to metabolize muscle-derived lactic acid (entering into the colon through the epithelial barrier) to produce the SCFA propionic acid, which improves endurance performance (Scheiman et al. 2019). In addition, in intestinal microbes-free mice, the colonization of intestinal microbiota resulted in a significant decrease in the expression of innate inflammatory mediators after transplantation of the stool of the excise mice group (containing the intestinal microbial flora), like leukocyte-1 $\beta$ (IL-1 $\beta$ ), indoleamine 2, 3 dioxygenase (IDO1) and interleukin-23 (IL-23). Further analysis revealed that there was a high proportion of specific microorganisms in the intestine (Allen et al. 2018), which are responsible for SCFA production, such as acetate, propionate and butyrate that have a beneficial effect on cardiometabolic health and intestinal cell survival (de la Cuesta-Zuluaga et al. 2018; Tye et al. 2018).

It was also demonstrated that the number of beneficial bacteria was significantly increased in the gut, and the levels of SCFAs were also increased by physical excise, particularly butyrate, a major substrate for colonocytes which represents the symbiosis between host and microbiota (Tye et al. 2018). Consistently, compared to healthy controls, gut microbial diversity of these rugby players was increased and enriched in the SCFA synthesis under execise and high protein diet (Barton et al. 2018). However, competitive cyclists had a higher abundance of Prevotella, which was negatively related to SCFA metabolism (Petersen et al. 2017). Moreover, fecal metabolites, including acetate, propionate and butyrate, are relatively increased in professional international rugby union athletes, which are associated with health benefit phenotypes (Barton et al. 2018).

In myocardial infarction (MI) mice, moderate intensity exercise was demonstrated to lead to intestinal microbial structural changes associated with cardiac functions, and two kinds of the bacteria Butyricimonas and Akkermansia were significantly enriched, which might play a beneficial role before the development of CVD (Liu et al. 2017). Similarly, it was also found that physical exercise was the most cost-effective lifestyle intervention to prevent and treat diabetes by altering gut microbiota, expecially Alistipes shahii, Alistipes putredinis, Ruminococcus gnavus, Eubacterium hallii and Coprococcus comes that may be related to the improvements in glucose homeostasis and insulin sensitivity (Liu et al. 2020). These findings render the feasibility of disease prevention via expanding the benefits of physical exercise and thus changing the gut microbiota. However, high-intensity exercise likely has a negative impact on gastrointestinal tract and leads to discomfort (Cresci and Bawden 2015), as evidenced by that $30 \%-90 \%$ distance runners have GI problems (de Oliveira, Burini and Jeukendrup 2014; Cresci and Bawden 2015).

\section{CHALLENGES AND PROSPECTS}

Gut microbiota play a vital role in human health. The development and use of intestinal flora-related methods and tools, including FMT, enterotype classification and innovations for gut bacterium culture, has broad prospects in the treatment of related diseases in future.

Although it has been clinically used in the treatment of gut microbe-orchestrating diseases, the long-term efficacy of FMT needs further monitoring. One of key issues is no uniform standard for the specific number of fecal bacteria used in FMT, leading to inconsistent clinical treatment results. Another is that FMT donors may have specific risk factors associated with a certain physical condition, such as metabolites, viruses and pathogenic bacteria that are normally difficult to be monitored. Therefore, it is urgent to establish a robust system to evaluate and supervise FMT from preparation to administration.

The simple classification of gut microbiota structures by gut enterotypes has a clinical potential, such as identifying an individual's disease situation and serving as a useful biomarker in disease progression. When we rely solely on gut enterotypes, the important microbial variations may be masked to some extent (Costea et al. 2018). It will be of high interest to investigate gut microbiota as a biomarker or index for age change, diet intake and disease progression, and for evaluation of the effects of probiotics, prebiotics and synbiotics (combination with prebiotic fiber) on clinical therapy.

Recently, a great progress has been made in culturing gut microbiota, but $23 \%-65 \%$ of microorganisms colonized in the human intestine cannot be cultured in vitro, which becomes an obstacle for exploring their biological functions (Strandwitz et al. 2019). The rise of omics analysis provides an opportunity to better understand the intestinal microbial flora. Using highresolution metagenomic sequencing, the key bacterial species as potential predictors have been identified in some common GI diseases, such as IBD and IBS (Vich Vila et al. 2018). However, the omics can not distinguish viable or relic DNA and in many human studies, only feces rather than intestines' digesta is collected. Therefore, exact microbiota dynamics is masked, which results in why most probiotics work well in the laboratory but not in clinical. It should be noted that potentially confounding factors of technique and biology have a mixed effect on the characteristics of intestinal microbiota markers, so further work is needed to solve the issues in exploring the influencing elements on gut microbiota (Sonnenburg and Backhed 2016; Kurilshikov et al. 2017; Jackson et al. 2018).

Measuring the direct interaction of the gut microbiota with food (components) also attracts more and more attention. Recent developments in this field include for instance usage of high tech telemetric capsules in humans, or non-invasive gut microbiota activity measurements using gas sensors detecting fermentation gasses in exhaled air [Nishijima et al. 2016]. The latter approach has been shown, using mouse models, to be able to quantitatively detect hydrogen levels in real-time, and an altered activity pattern within a few hours after switching from chow to a low digestible maize-starch diet, independent of total starch intake [Kuang et al. 2016; Fernandez-Calleja et al. 2018].

\section{SUPPLEMENTARY DATA}

Supplementary data are available at FEMSEC online. 


\section{AUTHOR CONTRIBUTIONS}

YZ conceived the study. JY, JW, YL, YeZ, XJ, WCC, EvS and YZ wrote the paper.

\section{FUNDING}

This work was financially supported by the National Natural Science Foundation of China (31472185 and U1703104) and the National Key Basic Research Program (973 program) of China (2015CB150300).

Conflicts of Interest. None declared.

\section{REFERENCES}

Adlerberth I, Strachan DP, Matricardi PM et al. Gut microbiota and development of atopic eczema in 3 European birth cohorts. J Allergy Clin Immunol 2007;120:343-50.

Allen JM, Mailing LJ, Cohrs J et al. Exercise training-induced modification of the gut microbiota persists after microbiota colonization and attenuates the response to chemically-induced colitis in gnotobiotic mice. Gut Microbes 2018;9:115-30.

An R, Wilms E, Masclee AAM et al. Age-dependent changes in GI physiology and microbiota: time to reconsider? Gut 2018;67:2213-22.

Arumugam M, Raes J, Pelletier E et al. Enterotypes of the human gut microbiome. Nature 2011;473:174-80.

Ayeni FA, Biagi E, Rampelli S et al. Infant and Adult Gut Microbiome and Metabolome in Rural Bassa and Urban Settlers from Nigeria. Cell Rep 2018;23:3056-67.

Bach JF. The hygiene hypothesis in autoimmunity: the role of pathogens and commensals. Nat Rev Immunol 2018;18: 105-20.

Barton W, Penney NC, Cronin O et al. The microbiome of professional athletes differs from that of more sedentary subjects in composition and particularly at the functional metabolic level. Gut 2018;67:625-33.

Batres-Marquez SP, Jensen HH, Upton J. Rice consumption in the United States: recent evidence from food consumption surveys. J Am Diet Assoc 2009;109:1719-27.

Bercik P, Collins SM, Verdu EF. Microbes and the gut-brain axis. Neurogastroenterol Motil 2012;24:405-13.

Bercik P, Denou E, Collins J et al. The intestinal microbiota affect central levels of brain-derived neurotropic factor and behavior in mice. Gastroenterology 2011;141:599-609, e1-3.

Bernstein CN. The brain-gut axis and stress in inflammatory bowel disease. Gastroenterol Clin North Am 2017;46:839-46.

Biagi E, Franceschi C, Rampelli S et al. Gut microbiota and extreme longevity. Curr Biol 2016;26:1480-5.

Biagi E, Rampelli S, Turroni S et al. The gut microbiota of centenarians: signatures of longevity in the gut microbiota profile. Mech Ageing Dev 2017;165:180-4.

Bian G, Gloor GB, Gong A et al. The gut microbiota of healthy aged Chinese is similar to that of the healthy young. mSphere 2017;2:e00327-17.

Bibbo S, Ianiro G, Gasbarrini A et al. Fecal microbiota transplantation: past, present and future perspectives. Minerva Gastroenterol Dietol 2017;63:420-30.

Botticelli A, Zizzari I, Mazzuca F et al. Cross-talk between microbiota and immune fitness to steer and control response to anti PD-1/PDL-1 treatment. Oncotarget 2017;8:8890-9.
Bowyer RCE, Jackson MA, Pallister T et al. Use of dietary indices to control for diet in human gut microbiota studies. Microbiome 2018;6:77.

Brewster R, Tamburini FB, Asiimwe E et al. Surveying Gut Microbiome Research in Africans: toward Improved Diversity and Representation. Trends Microbiol 2019;27:824-35.

Bruce-Keller AJ, Salbaum JM, Luo M et al. Obese-type gut microbiota induce neurobehavioral changes in the absence of obesity. Biol Psychiatry 2015;77:607-15.

Brunkwall L, Orho-Melander M. The gut microbiome as a target for prevention and treatment of hyperglycaemia in type 2 diabetes: from current human evidence to future possibilities. Diabetologia 2017;60:943-51.

Buford TW. (Dis)Trust your gut: the gut microbiome in age-related inflammation, health, and disease. Microbiome 2017;5:80.

Candon S, Perez-Arroyo A, Marquet C et al. Antibiotics in early life alter the gut microbiome and increase disease incidence in a spontaneous mouse model of autoimmune insulindependent diabetes. PLoS One 2015;10:e0125448.

Capurso G, Lahner E. The interaction between smoking, alcohol and the gut microbiome. Best Pract Res Clin Gastroenterol 2017;31:579-88.

Carbonetto B, Fabbro MC, Sciara M et al. Human Microbiota of the Argentine Population- A Pilot Study. Front Microbiol 2016;7:51.

Charbonneau MR, O'Donnell D, Blanton LV et al. Sialylated Milk Oligosaccharides Promote Microbiota-Dependent Growth in Models of Infant Undernutrition. Cell 2016;164:859-71.

Costea PI, Hildebrand F, Arumugam M et al. Enterotypes in the landscape of gut microbial community composition. Nat Microbiol 2018;3:8-16.

Cox LM, Yamanishi S, Sohn J et al. Altering the intestinal microbiota during a critical developmental window has lasting metabolic consequences. Cell 2014;158:705-21.

Cresci GA, Bawden E. Gut Microbiome: what We Do and Don't Know. Nutr Clin Pract 2015;30:734-46.

David LA, Maurice CF, Carmody $\mathrm{RN}$ et al. Diet rapidly and reproducibly alters the human gut microbiome. Nature 2014;505:559-63.

De Filippis F, Pellegrini N, Vannini L et al. High-level adherence to a Mediterranean diet beneficially impacts the gut microbiota and associated metabolome. Gut 2016;65:1812-21.

de la Cuesta-Zuluaga J, Mueller NT, Alvarez-Quintero R et al. Higher Fecal Short-Chain Fatty Acid Levels Are Associated with Gut Microbiome Dysbiosis, Obesity, Hypertension and Cardiometabolic Disease Risk Factors. Nutrients 2018;11:51.

de Oliveira EP, Burini RC, Jeukendrup A. Gastrointestinal complaints during exercise: prevalence, etiology, and nutritional recommendations. Sports Med 2014;44 Suppl 1:S79-85.

de Weerth C. Do bacteria shape our development? Crosstalk between intestinal microbiota and HPA axis. Neurosci Biobehav Rev 2017;83:458-71.

Dinan TG, Cryan JF. The Microbiome-Gut-Brain Axis in Health and Disease. Gastroenterol Clin North Am 2017b;46:77-89.

Elkrief A, Derosa L, Zitvogel L et al. The intimate relationship between gut microbiota and cancer immunotherapy. Gut Microbes 2018:1-5.

Escobar JS, Klotz B, Valdes BE et al. The gut microbiota of Colombians differs from that of Americans, Europeans and Asians. BMC Microbiol 2014;14:311.

Fernandez-Calleja JMS, Konstanti P, Swarts HJM et al. Noninvasive continuous real-time in vivo analysis of microbial hydrogen production shows adaptation to fermentable carbohydrates in mice. Sci Rep 2018;8:15351. 
Fontana L, Partridge L. Promoting health and longevity through diet: from model organisms to humans. Cell 2015;161:106-18.

Forslund K, Hildebrand F, Nielsen T et al. Corrigendum: disentangling type 2 diabetes and metformin treatment signatures in the human gut microbiota. Nature 2017;545:116.

Foster JA, McVey Neufeld KA. Gut-brain axis: how the microbiome influences anxiety and depression. Trends Neurosci 2013;36:305-12.

Gentile CL, Weir TL. The gut microbiota at the intersection of diet and human health. Science 2018;362:776-80.

Gibbons SM, Kearney SM, Smillie CS et al. Two dynamic regimes in the human gut microbiome. PLoS Comput Biol 2017;13:e1005364.

Guilleminault L, Williams EJ, Scott HA et al. Diet and Asthma: is It Time to Adapt Our Message? Nutrients 2017;9.

Gupta A, Cifu AS, Khanna S. Diagnosis and Treatment of Clostridium difficile Infection. JAMA 2018;320:1031-2.

Hansen MEB, Rubel MA, Bailey AG et al. Population structure of human gut bacteria in a diverse cohort from rural Tanzania and Botswana. Genome Biol 2019;20:16.

Holscher HD. Dietary fiber and prebiotics and the gastrointestinal microbiota. Gut Microbes 2017;8:172-84.

Ho NT, Li F, Lee-Sarwar KA et al. Meta-analysis of effects of exclusive breastfeeding on infant gut microbiota across populations. Nat Commun 2018;9:4169.

Ianiro G, Tilg H, Gasbarrini A. Antibiotics as deep modulators of gut microbiota: between good and evil. Gut 2016;65:1906-15.

Iizumi T, Battaglia T, Ruiz V et al. Gut Microbiome and Antibiotics. Arch Med Res 2017;48:727-34.

Jackson MA, Goodrich JK, Maxan ME et al. Proton pump inhibitors alter the composition of the gut microbiota. Gut 2016;65: 749-56.

Jackson MA, Verdi S, Maxan ME et al. Gut microbiota associations with common diseases and prescription medications in a population-based cohort. Nat Commun 2018;9:2655.

JanssenDuijghuijsen LM, Mensink M, Lenaerts K et al. The effect of endurance exercise on intestinal integrity in well-trained healthy men. Physiol Rep 2016;4:e12994.

Jiang H, Ling Z, Zhang Y et al. Altered fecal microbiota composition in patients with major depressive disorder. Brain Behav Immun 2015;48:186-94.

Kieffer DA, Martin RJ, Adams SH. Impact of Dietary Fibers on Nutrient Management and Detoxification Organs: gut, Liver, and Kidneys. Adv Nutr 2016;7:1111-21.

Kim S, Covington A, Pamer EG. The intestinal microbiota: antibiotics, colonization resistance, and enteric pathogens. Immunol Rev 2017;279:90-105.

Kong F, Deng F, Li Y et al. Identification of gut microbiome signatures associated with longevity provides a promising modulation target for healthy aging. Gut Microbes 2018:1-6.

Kong F, Hua Y, Zeng B et al. Gut microbiota signatures of longevity. Curr Biol 2016;26:R832-R3.

Kuang YS, Li SH, Guo Y et al. Composition of gut microbiota in infants in China and global comparison. Sci Rep 2016;6:36666.

Kumbhare SV, Kumar H, Chowdhury SP et al. A cross-sectional comparative study of gut bacterial community of Indian and Finnish children. Sci Rep 2017;7:10555.

Kurilshikov A, Wijmenga C, Fu J et al. Host Genetics and Gut Microbiome: challenges and Perspectives. Trends Immunol 2017;38:633-47.

Lach G, Schellekens H, Dinan TG et al. Anxiety, Depression, and the Microbiome: a Role for Gut Peptides. Neurotherapeutics 2018;15:36-59.
Lam YY, Zhang C, Zhao L. Causality in dietary interventionsbuilding a case for gut microbiota. Genome Med 2018;10:62.

Levy M, Kolodziejczyk AA, Thaiss CA et al. Dysbiosis and the immune system. Nat Reu Immunol 2017;17:219-32.

Liao M, Xie Y, Mao Y et al. Comparative analyses of fecal microbiota in Chinese isolated Yao population, minority Zhuang and rural Han by 16sRNA sequencing. Sci Rep 2018; 8:1142.

Liu RT. The microbiome as a novel paradigm in studying stress and mental health. Am Psychol 2017;72:655-67.

Liu S, da Cunha AP, Rezende RM et al. The Host Shapes the Gut Microbiota via Fecal MicroRNA. Cell Host Microbe 2016;19: 32-43.

Liu Y, Wang Y, Ni Y et al. Gut Microbiome Fermentation Determines the Efficacy of Exercise for Diabetes Prevention. Cell Metab 2020:77-91.e5.

Liu Z, Liu HY, Zhou $\mathrm{H}$ et al. Moderate-Intensity Exercise Affects Gut Microbiome Composition and Influences Cardiac Function in Myocardial Infarction Mice. Front Microbiol 2017;8:1687.

Magne F, O'Ryan ML, Vidal R et al. The human gut microbiome of Latin America populations: a landscape to be discovered. Curr Opin Infect Dis 2016;29:528-37.

Maier L, Pruteanu M, Kuhn M et al. Extensive impact of nonantibiotic drugs on human gut bacteria. Nature 2018;555: 623-8.

Mano F, Ikeda K, Joo E et al. The Effect of White Rice and White Bread as Staple Foods on Gut Microbiota and Host Metabolism. Nutrients 2018;10.

Maruvada P, Leone V, Kaplan LM et al. The Human Microbiome and Obesity: moving beyond Associations. Cell Host Microbe 2017;22:589-99.

McDonald JAK, Mullish BH, Pechlivanis A et al. Inhibiting Growth of Clostridioides difficile by Restoring Valerate, Produced by the Intestinal Microbiota. Gastroenterology 2018;155:1495-507 e15.

McKenzie C, Tan J, Macia L et al. The nutrition-gut microbiomephysiology axis and allergic diseases. Immunol Rev 2017;278:277-95.

Milani C, Duranti S, Bottacini F et al. The First Microbial Colonizers of the Human Gut: composition, Activities, and Health Implications of the Infant Gut Microbiota. Microbiol Mol Biol Rev 2017;81.

Mitsou EK, Kakali A, Antonopoulou S et al. Adherence to the Mediterranean diet is associated with the gut microbiota pattern and gastrointestinal characteristics in an adult population. Br J Nutr 2017;117:1645-55.

Mohammadkhah AI, Simpson EB, Patterson SG et al. Development of the Gut Microbiome in Children, and Lifetime Implications for Obesity and Cardiometabolic Disease. Children (Basel) 2018;5:160.

Mullish BH, Quraishi MN, Segal JP et al. The use of faecal microbiota transplant as treatment for recurrent or refractory Clostridium difficile infection and other potential indications: joint British Society of Gastroenterology (BSG) and Healthcare Infection Society (HIS) guidelines. Gut 2018;67:1920-41.

Munyaka PM, Khafipour E, Ghia JE. External influence of early childhood establishment of gut microbiota and subsequent health implications. Front Pediatr 2014;2:109.

Ni Lochlainn M, Bowyer RCE, Steves CJ. Dietary Protein and Muscle in Aging People: the Potential Role of the Gut Microbiome. Nutrients 2018;10. 
Nishijima S, Suda W, Oshima $\mathrm{K}$ et al. The gut microbiome of healthy Japanese and its microbial and functional uniqueness. DNA Res 2016;23:125-33.

O'Toole PW, Jeffery IB. Gut microbiota and aging. Science 2015;350:1214-5.

Okubo R, Koga $\mathrm{M}$, Katsumata $\mathrm{N}$ et al. Effect of bifidobacterium breve A-1 on anxiety and depressive symptoms in schizophrenia: a proof-of-concept study. J Affect Disord 2018;245:377-85.

Ottman N, Smidt H, de Vos WM et al. The function of our microbiota: who is out there and what do they do? Front Cell Infect Microbiol 2012;2:104.

Pacheco AR, Curtis MM, Ritchie JM et al. Fucose sensing regulates bacterial intestinal colonization. Nature 2012;492:113-7.

Pannaraj PS, Li F, Cerini C et al. Association Between Breast Milk Bacterial Communities and Establishment and Development of the Infant Gut Microbiome. JAMA Pediatr 2017;171:647-54.

Petersen LM, Bautista EJ, Nguyen $\mathrm{H}$ et al. Community characteristics of the gut microbiomes of competitive cyclists. Microbiome 2017;5:98.

Pignanelli M, Just C, Bogiatzi C et al. Mediterranean Diet Score: associations with Metabolic Products of the Intestinal Microbiome, Carotid Plaque Burden, and Renal Function. Nutrients 2018;10:779.

Rautava S. Early microbial contact, the breast milk microbiome and child health. J Dev Orig Health Dis 2016;7:5-14.

Rehman A, Rausch P, Wang J et al. Geographical patterns of the standing and active human gut microbiome in health and IBD. Gut 2016;65:238-48.

Riaz Rajoka MS, Zhao H, Li N et al. Origination, change, and modulation of geriatric disease-related gut microbiota during life. Appl Microbiol Biotechnol 2018;102:8275-89.

Robertson RC, Manges AR, Finlay BB et al. The Human Microbiome and Child Growth - First 1000 Days and Beyond. Trends Microbiol 2019;27:131-47.

Rothschild D, Weissbrod O, Barkan E et al. Environment dominates over host genetics in shaping human gut microbiota. Nature 2018;555:210-5.

Routy B, Le Chatelier E, Derosa L et al. Gut microbiome influences efficacy of PD-1-based immunotherapy against epithelial tumors. Science 2018;359:91-7.

Ruengsomwong S, La-Ongkham O, Jiang J et al. Microbial Community of Healthy Thai Vegetarians and Non-Vegetarians, Their Core Gut Microbiota, and Pathogen Risk. J Microbiol Biotechnol 2016;26:1723-35.

Rutayisire E, Huang K, Liu Y et al. The mode of delivery affects the diversity and colonization pattern of the gut microbiota during the first year of infants' life: a systematic review. BMC Gastroenterol 2016;16:86.

Salazar N, Arboleya S, Valdes L et al. The human intestinal microbiome at extreme ages of life. Dietary intervention as a way to counteract alterations. Front Genet 2014;5:406.

Salazar N, Valdes-Varela L, Gonzalez S et al. Nutrition and the gut microbiome in the elderly. Gut Microbes 2017;8:82-97.

Sarkar A, Harty S, Lehto SM et al. The Microbiome in Psychology and Cognitive Neuroscience. Trends Cogn Sci 2018;22:611-36.

Scheiman J, Luber JM, Chavkin TA et al. Meta-omics analysis of elite athletes identifies a performance-enhancing microbe that functions via lactate metabolism. Nat Med 2019;25: 1104-9.

Sivan A, Corrales L, Hubert $\mathrm{N}$ et al. Commensal Bifidobacterium promotes antitumor immunity and facilitates anti-PD-L1 efficacy. Science 2015;350:1084-9.

Song M, Chan AT. The Potential Role of Exercise and Nutrition in Harnessing the Immune System to Improve Colorectal Cancer Survival. Gastroenterology 2018;155:596-600.

Sonnenburg JL, Backhed F. Diet-microbiota interactions as moderators of human metabolism. Nature 2016;535:56-64.

Soularue E, Lepage P, Colombel JF et al. Enterocolitis due to immune checkpoint inhibitors: a systematic review. Gut 2018;67:2056-67.

Stokholm J, Sevelsted A, Bonnelykke K et al. Maternal propensity for infections and risk of childhood asthma: a registry-based cohort study. Lancet Respir Med 2014;2:631-7.

Strandwitz P, Kim KH, Terekhova D et al. GABA-modulating bacteria of the human gut microbiota. Nat Microbiol 2019: 396-403.

Tai N, Wong FS, Wen L. The role of gut microbiota in the development of type 1, type 2 diabetes mellitus and obesity. Rev Endocr Metab Disord 2015;16:55-65.

Tandon D, Haque MM, R S et al. A snapshot of gut microbiota of an adult urban population from Western region of India. PLoS One 2018;13:e0195643.

Tang WHW, Li DY, Hazen SL. Dietary metabolism, the gut microbiome, and heart failure. Nat Rev Cardiol 2018:137-54.

Tang WHW, Li DY, Hazen SL. Dietary metabolism, the gut microbiome, and heart failure. Nat Reu Cardiol 2019;16:137-54.

Tetel MJ, de Vries GJ, Melcangi RC et al. Steroids, stress and the gut microbiome-brain axis. J Neuroendocrinol 2018;30:12548.

Ticinesi A, Tana C, Nouvenne A. The intestinal microbiome and its relevance for functionality in older persons. Curr Opin Clin Nutr Metab Care 2019;22:4-12.

Tye $\mathrm{H}$, Yu CH, Simms LA et al. NLRP1 restricts butyrate producing commensals to exacerbate inflammatory bowel disease. Nat Commun 2018;9:3728.

Vanegas SM, Meydani M, Barnett JB et al. Substituting whole grains for refined grains in a 6 -wk randomized trial has a modest effect on gut microbiota and immune and inflammatory markers of healthy adults. Am J Clin Nutr 2017;105: 635-50.

Vich Vila A, Imhann F, Collij V et al. Gut microbiota composition and functional changes in inflammatory bowel disease and irritable bowel syndrome. Sci Transl Med 2018;10:eaap8914.

Vindigni SM, Surawicz CM. Fecal Microbiota Transplantation. Gastroenterol Clin North Am 2017;46:171-85.

Weersma RK, Zhernakova A, Fu J. Interaction between drugs and the gut microbiome. Gut 2020;69:1510-9.

Winglee K, Howard AG, Sha W et al. Recent urbanization in China is correlated with a Westernized microbiome encoding increased virulence and antibiotic resistance genes. Microbiome 2017:5:121.

Wypych TP, Marsland BJ, Ubags NDJ. The Impact of Diet on Immunity and Respiratory Diseases. Ann Am Thorac Soc 2017;14:S339-S47.

Yi M, Yu S, Qin S et al. Gut microbiome modulates efficacy of immune checkpoint inhibitors. J Hematol Oncol 2018;11:47. 